\title{
Metodología docente y de evaluación en el nuevo espacio de convergencia europea para la formación del docente de Educación Secundaria
}

\author{
Teaching and assessment methodology in the new space of European convergence \\ in the training of teachers of Secondary Education
Metodologia docente e de avaliação em um novo espaço de convergência europeia para a formação de professores do Ensino Secundário

\section{$M^{a}$ del Carmen Pegalajar Palomino}

Universidad Católica de Murcia, mdcpegalajar@ucam.edu

\begin{abstract}
RESUMEN
Este estudio pretende examinar las percepciones del alumnado del Máster en formación del profesorado en Educación Secundaria Obligatoria hacia la metodología docente y evaluativa del profesorado universitario. Para ello, se ha utilizado un diseño descriptivo-correlacional llevándose a cabo la recogida de datos mediante la adaptación del "Cuestionario para la Evaluación de la Metodología Docente y Evaluativa de los Profesores Universitarios". Los resultados demuestran cómo el profesorado no sólo utiliza el examen como instrumento de evaluación; el alumnado entiende que un buen profesor es aquel que explica adecuadamente su asignatura y domina la materia que imparte. Además, se valora el que éste realice aplicaciones de la teoría a la práctica en el aula y fomente la participación del alumnado. También, el alumnado aprecia que el profesor realice una síntesis de lo tratado en las tutorías, planifique su asignatura adecuadamente y se interese por él a nivel personal.
\end{abstract}

Palabras clave: metodología docente, evaluación, formación, Máster, percepciones.

\section{ABSTRACT}

This study seeks to examine the perceptions of students of the Master in Teacher Education in Secondary Education about the teaching and assessment methodology of university teachers. A descriptive co-relational design was used, carrying out data collection by adapting the "Questionnaire for the Assessment of Teaching Methodology and Evaluative University Professors." Results show that teachers use the test as an assessment tool; students understand that an excellent teacher adequately explains its subject and dominates the subject they teach. In addition, applications of the theory to classroom practice and encouragement of students' participation is appreciated. Also, students distinguish teachers making summaries of what it was discussed in the tutorials, preparing the planning of their subjects properly and showing interest in them at a personal level.

Keywords: teaching methodology, assessment, training, Master, perceptions.

\section{RESUMO}

Pretende-se analisar as percepções dos estudantes do Mestrado em Formação de Professores no Ensino Secundário Obrigatório para a metodologia docente e avaliativa dos professores universitários. Para isso, foi utilizado um desenho descritivo- correlacional, levando-se em conta a coleta de dados mediante a adaptação do "Questionário para a Avaliação da Metodologia Docente e Avaliativa dos Professores Universitários.” Resultados demonstram como os professores não apenas utilizam o exame como instrumento de avaliação; os estudantes entendem que um bom professor é aquele que explica adequadamente seu conteúdo e domina a matéria que lhes ensinam. Além disso, avalia-se que sejam realizadas aplicações da teoria em aulas práticas e que seja incentivada a participação 
Estudios Pedagógicos XLII, N 1: 209-222, 2016

METODOLOGÍA DOCENTEY DE EVALUACIÓN EN EL NUEVO ESPACIO DE CONVERGENCIA EUROPEA PARA LA FORMACIÓN DEL DOCENTE DE EDUCACIÓN SECUNDARIA

dos estudantes nas aulas. Os estudantes também observam que o professor faça um resumo do que foi discutido nas aulas, planeje adequadamente o assunto e que tenha interesse por ele.

Palavras-chave: metodologia docente, avaliação, formação, Mestrado, percepções.

\section{INTRODUCCIÓN}

Existe un consenso generalizado para reconocer la importancia de los procesos educativos para el desarrollo de las personas y las sociedades. Un punto sustantivo para promover la calidad educativa es cómo ocurre el ejercicio docente en el aula, ya que buena parte del resultado deriva de cómo se ejerce la enseñanza (Tirado, Miranda y Sánchez, 2007). Lejos de ser una actividad simplista que cualquiera pueda realizar, la enseñanza es un acto complejo y multidimensional, por ser una tarea en la que de manera propositiva y deliberada se pretende que alguien aprenda de otro (Guzmán, 2009).

En el sistema universitario, y dado el actual proceso de convergencia europea, se fomenta una visión interdisciplinar de la enseñanza, favoreciendo una formación que considere las actitudes y competencias genéricas a adquirir por el alumnado, con implicaciones directas en las metodologías docentes (González y García; 2007; Ion y Cano, 2011; Palomares, 2011) y en los procedimientos de evaluación (Carrasco et al., 2010; Cid, Zabalza y Doval, 2012; Zabalza, 2012).

Para ello, se requiere de un sistema de aprendizaje autónomo y tutorizado que lleve al alumnado a construir e interpretar el entorno de manera significativa (Gairín, Feixas, Guillamón y Quinquer, 2004; Herrera y Cabo, 2008) mediante un aprendizaje a lo largo de toda la vida (Herrera, Lorenzo y Rodríguez, 2008; Méndez, 2005) que favorezca el máximo desarrollo de sus capacidades y, por lo tanto, una mejora en su proceso de crecimiento y desarrollo integral (Kallioinen, 2010).

Además, se ha trasladado el centro de interés hacia la actividad del alumnado y su aprendizaje, frente al énfasis de la enseñanza impartida por el profesor (Padilla y Gil, 2008). De este modo, el estudiante debe ser un sujeto implicado de manera activa en su proceso de aprendizaje (Gargallo, Suárez y Ferreras, 2007; Pozo y Pérez, 2009), mientras que el profesor universitario debe posibilitar, facilitar y guiar al alumnado para acceder intelectualmente a los contenidos y prácticas profesionales de una determinada disciplina (Herrera, 2007; Moreno, Bajo, Moya, Maldonado y Tudela, 2007; Sander, 2005).

Mas (2011) propone una serie de competencias que debe poseer el profesorado universitario para el desarrollo de su función docente con garantías de calidad tales como: diseñar la guía docente de acuerdo a las necesidades, el contexto y el perfil profesional con coordinación de otros profesionales; desarrollar el proceso de enseñanza-aprendizaje, propiciando oportunidades de aprendizaje (a nivel individual y grupal); tutorizar el proceso de aprendizaje del alumnado, propiciando acciones que le permitan una mayor autonomía; evaluar el proceso de enseñanza-aprendizaje; contribuir activamente a la mejora de la docencia y participar activamente en la dinámica académico-organizativa de la institución. De igual modo, el autor alude a la preocupación del docente por desarrollar su función investigadora, crear conocimiento y mejorar su campo científico, ofrecer nuevas propuestas metodológicas adaptadas al alumnado y la materia, innovar en su realidad y en su contexto, etc.

Autores como Palazón, Gómez, Gómez, Pérez y Gómez (2011) y Rodríguez-Martín y Álvarez-Arregui (2013) destacan una preferencia del alumnado hacia el desarrollo de 
metodologías activas y participativas para favorecer la motivación, el desarrollo de proyectos y aprender a autogestionarse en el trabajo individual y en equipo (Hernández-Pina, 2003; Martínez et al., 2007). La investigación desarrollada por Hernández-Amorós y Carrasco (2012) revela cómo el alumnado del Máster de Secundaria no se muestra satisfecho con el tiempo establecido para su formación didáctica, la vinculación entre teoría y práctica en el desarrollo de programas formativos, así como con la coordinación entre el profesorado, manifestando haberse desarrollado acciones excesivamente individualistas.

Así pues, Sáez y Ruiz (2012) avisan de la persistencia de enfoques tradicionales en la docencia universitaria, destacando la necesidad de aprovechar técnicas orientadas al aprendizaje activo, trabajo colaborativo entre grupos, adquisición de habilidades para resolver problemas y posibilitar que el alumnado desarrolle un pensamiento crítico, capaz de comprender y valorar la información disponible en el contexto actual. Además, autores como Medina, Domínguez y Ribeiro (2011) revelan cómo el profesorado universitario ha realizado una docencia orientada al saber académico, siendo ésta insuficiente para dar respuesta a los retos que plantea la actual sociedad del conocimiento, las demandas laborales y los cambios en los estilos y modos de vida laboral.

Se precisa, pues, de un giro metodológico en la enseñanza, en el que tenga cabida el desarrollo de experiencias de aprendizaje atractivas e integradas; dicho enfoque requiere la reducción de sesiones tradicionales y presenciales centradas en la adquisición de conocimientos conceptuales en el alumnado (Cano, 2008). Para ello, Armengol et al. (2009) apuestan por el diseño de programas de formación de acceso y permanente para los docentes universitarios en el manejo de metodologías activas.

Teniendo en cuenta esta situación, cobra especial importancia el estudio de las potencialidades y debilidades del profesorado universitario de este título en cuanto al "cómo enseñar" en el proceso de enseñanza-aprendizaje, estableciendo propuestas de mejora que favorezcan la calidad de la Educación Superior.

\section{DISEÑO METODOLÓGICO}

Este estudio pretende analizar las percepciones del alumnado del Máster en formación del profesorado de Educación Secundaria Obligatoria acerca de la metodología docente y evaluativa del profesorado. Para ello, se ha utilizado una metodología cuantitativa basada en el método descriptivo-correlacional, pues se considera que es la adecuada cuando se desea hacer una exploración de un determinado fenómeno educativo para, a continuación, conocer esta realidad y poder mejorarla.

De manera más concreta, esta investigación pretende:

- Conocer las percepciones del alumnado acerca de la concepción tradicional del conocimiento y la enseñanza del profesorado en la Educación Superior.

- Examinar las valoraciones del alumnado acerca de la concepción activa y constructivista del conocimiento y del aprendizaje del profesorado y su incidencia en el aula universitaria.

- Examinar las habilidades docentes del profesorado universitario de posgrado.

- Determinar el grado de relación entre las percepciones del alumnado acerca de la concepción tradicional de la enseñanza, la concepción activa del conocimiento y las habilidades docentes. 


\subsection{INSTRUMENTO DE RECOGIDA DE DATOS}

Para la recogida de datos, ha sido necesaria la adaptación del cuestionario "CEMEDEPU" (Cuestionario para la Evaluación de la Metodología Docente y Evaluativa de los Profesores Universitarios) elaborado por Gargallo, Suárez, Gargella y Fernández (2011). Se trata de una escala dirigida al alumnado universitario; organizada en tres escalas, consta de 48 ítems cuyas opciones de respuesta oscilan entre 1 y 5 (siendo $1=$ muy en desacuerdo y $5=$ muy de acuerdo).

Dicho instrumento ha sido elaborado a partir del modelo centrado en la enseñanza y en el aprendizaje, así como la concreción de las habilidades docentes del profesor universitario competente. Para la adaptación del cuestionario a la población objeto de estudio, se han modificado cada uno de los ítems, dirigiéndoles las preguntas al alumnado aunque cuidando que no se modificase el contenido del mismo.

Así pues, se ha comprobado la validez de contenido mediante un juicio de expertos y la aplicación de una prueba piloto. En el primer caso, se solicitó a varios profesores universitarios, especialistas en el área de Didáctica y Organización Escolar de varias universidades españolas, que llevasen a cabo una valoración global del instrumento, indicando en una escala de 0-10 la adecuación de la información inicial. En relación a los ítems, y al tratarse de un cuestionario adaptado, los jueces debían indicar el grado de pertenencia de cada ítem al objeto de estudio (contenido) y el grado de precisión y adecuación del mismo (forma).

A continuación, se realizó la interpretación de las respuestas aportadas por cada uno de los expertos, los cuáles dieron lugar a la modificación de algunos de los ítems para facilitar la comprensión de los mismos por el alumnado. En líneas generales, las evaluaciones de los expertos mostraron que la mayoría de los ítems eran correctos y adecuados, en comprensión y escritura, a los destinatarios del cuestionario.

Una vez realizado el juicio de expertos, y con la intención de conocer el grado de comprensión de los ítems del cuestionario por parte del alumnado y analizar la validez de constructo, se procedió a la aplicación de una prueba piloto a 100 estudiantes del Máster en formación del profesorado de la Universidad de Jaén. Así pues, el índice de adecuación muestral KMO alcanza un valor de .914 y la prueba de esfericidad de Bartlett es de $6038.066(\mathrm{p}=.000)$. Además, se ha realizado un análisis factorial exploratorio confirmatorio, revelándose la existencia de tres factores que explican el $49.03 \%$ de la varianza.

El primer factor, compuesto por 13 ítems, está centrado en el modelo basado en la enseñanza y recoge aspectos referidos a la concepción tradicional del conocimiento y del aprendizaje, concepción tradicional de la enseñanza y del papel del profesor y uso de métodos de enseñanza y evaluación tradicionales.

El segundo factor, de 15 ítems, se basa en el modelo centrado en el aprendizaje y recoge aspectos tales como la concepción activa y constructiva de la enseñanza y actuación coherente, el conocimiento como construcción, interacción eficaz con el alumnado en tutorías, seminarios, uso de las TICs y de metodologías de evaluación formativa.

La tercera escala, de habilidades docentes, consta de 20 ítems que analizan aspectos como las habilidades de planificación/información al alumnado, manejo instruccional, interacción/relación educativa y evaluación. 
Por su parte, y para determinar la fiabilidad del instrumento se ha utilizado el coeficiente alfa de Cronbach, obteniéndose resultados bastante satisfactorios (.956). Además, y para el método de las dos mitades, se ha obtenido para la primera parte, un valor de .896 y, para la segunda, de .949 , lo que indica que la escala es muy confiable. Por otro lado, el coeficiente de Spearman-Brown arroja un valor de .870, lo cual induce a considerar la alta fiabilidad del instrumento.

\subsection{MUESTRA}

La población objeto de estudio está compuesta por 264 estudiantes del Máster Universitario en Formación del Profesorado en Educación Secundaria Obligatoria y Bachillerato, Formación Profesional y Enseñanza de Idiomas de la Universidad Católica de Murcia, que durante el curso 2013/14 realizan sus estudios universitarios. Para la selección de los sujetos se ha utilizado un muestreo casual o incidental, de forma que se ha adscrito a la muestra aquellos sujetos que al azar respondieron el cuestionario $(n=252)$. Para el cálculo de la muestra participante, se ha utilizado la fórmula para poblaciones con menos de 100.000 sujetos, con un nivel de confianza del 95\% y un error de estimación máximo de $4 \%$.

De la muestra, un $59.6 \%$ son mujeres, frente al $40.4 \%$ que está compuesta por hombres. Sus edades oscilan, de manera mayoritaria, entre 21 y 25 años (42.9\%), y entre 26 y 30 años (33.7\%), aunque un porcentaje algo menor (23.4\%) tiene una edad superior a los 30 años. En cuanto a la especialidad por la que cursan dicho título, un $32.7 \%$ lo realiza desde el área de Humanidades, Ciencias Sociales y de la Empresa, un 27.5\% por Ciencia y Tecnología, un $20.3 \%$ por Actividad Física y del Deporte, un $17.5 \%$ por Filología e Idiomas y, finalmente, un $2.0 \%$ por Artes. El motivo por el que han accedido a este título de Posgrado es, para un $75.7 \%$ de los casos, para poder ser profesor de Educación Secundaria, Formación Profesional, Bachillerato o Idiomas; un 14.8\% de los estudiantes se han matriculado para acceder a estudios de Doctorado y, finalmente, un $9.5 \%$ para obtener una formación psicopedagógica complementaria.

\subsection{PROCEDIMIENTO}

La cumplimentación del cuestionario por parte de la muestra se ha llevado a cabo a partir de Noviembre de 2013, fecha en la que el alumnado del Máster en Formación del Profesorado se encontraba realizando el módulo genérico del título. Éste presenta un carácter semipresencial para el alumnado, debiendo acudir a la Universidad para la realización de tutorías personales y exámenes para cada una de las asignaturas.

\subsection{ANÁLISIS DE DATOS}

Para el análisis de datos, se ha utilizado el paquete informático Statistickal Package for Social Sciences (SPSS, versión 21) al considerarlo un recurso idóneo para esta investigación. Así pues, se ha llevado a cabo un análisis descriptivo de cada uno de los ítems que conforman las tres escalas del cuestionario. Además, y con la intención de conocer el grado de relación entre los factores que conforman las escalas del cuestionario, se ha utilizado el coeficiente de correlación de Pearson. 
Estudios Pedagógicos XLII, N 1: 209-222, 2016

METODOLOGIA DOCENTEY DE EVALUACIÓN EN EL NUEVO ESPACIO DE CONVERGENCIA EUROPEA PARA LA FORMACIÓN DEL DOCENTE DE EDUCACIÓN SECUNDARIA

\section{RESULTADOS}

\subsection{MODELO CENTRADO EN LA ENSEÑANZA}

El alumnado de posgrado afirma cómo el profesorado no sólo hace uso de pruebas objetivas para la evaluación del alumnado. Asimismo, entiende que dicho proceso no debe limitarse a la simple valoración de los conocimientos adquiridos. Para él, un buen profesor es aquel que explica adecuadamente los contenidos de su asignatura y domina la materia que imparte; debe ser responsable de organizar y saber trasmitir los contenidos al alumnado, no siendo la lección magistral y la recogida de apuntes la metodología fundamental.

De igual modo, afirma que el profesorado define el aprendizaje como un proceso tendente a incrementar los conocimientos de que dispone el alumnado sobre una determinada materia, no debiendo reducirse la formación universitaria al aprendizaje y comprensión de los contenidos científicos de las asignaturas.

Tabla 1. Análisis descriptivo para la escala "Modelo centrado en la enseñanza"

\begin{tabular}{|l|c|c|}
\hline \multicolumn{1}{|c|}{ Ítem } & Media & Desv. Típica \\
\hline El único método de evaluación son los exámenes & 2.64 & 1.335 \\
\hline $\begin{array}{l}\text { Al profesorado le basta con que los alumnos aprendan los } \\
\text { contenidos científicos fundamentales de la asignatura }\end{array}$ & 2.82 & 1.157 \\
\hline $\begin{array}{l}\text { El profesorado entiende que la evaluación debe limitarse a la } \\
\text { valoración de los conocimientos adquiridos }\end{array}$ & 2.90 & 1.168 \\
\hline El mejor método de evaluación es el examen & 2.98 & 1.150 \\
\hline $\begin{array}{l}\text { Mi papel en las tutorías presenciales es estar atento y tomar } \\
\text { bien los apuntes }\end{array}$ & 3.28 & 1.134 \\
\hline $\begin{array}{l}\text { El trabajo esencial del profesor universitario es transmitir los } \\
\text { conocimientos a sus alumnos }\end{array}$ & 3.34 & 1.019 \\
\hline $\begin{array}{l}\text { En las tutorías presenciales, la lección magistral es la } \\
\text { metodología fundamental }\end{array}$ & 3.35 & 1.070 \\
\hline $\begin{array}{l}\text { El profesorado utiliza como método de evaluación los } \\
\text { exámenes con formato de prueba objetiva }\end{array}$ & 3.41 & .912 \\
\hline $\begin{array}{l}\text { Lo más importante de un buen profesor es dominar la } \\
\text { materia que imparte }\end{array}$ & 3.61 & .960 \\
\hline Un buen profesor es el que explica bien su asignatura & 3.67 & .965 \\
\hline $\begin{array}{l}\text { El profesorado debe aprovechar el tiempo de las tutorías } \\
\text { presenciales para explicar bien los contenidos de la } \\
\text { asignatura }\end{array}$ & 3.71 & 1.021 \\
\hline $\begin{array}{l}\text { El profesorado entiende que el aprendizaje supone } \\
\text { incrementar los conocimientos de que dispone el alumnado }\end{array}$ & 3,79 & .951 \\
\hline $\begin{array}{l}\text { La responsabilidad fundamental del profesor es organizar } \\
\text { bien los conocimientos que deben aprender los alumnos }\end{array}$ & 3,81 & .947 \\
\hline
\end{tabular}




\subsection{MODELO CENTRADO EN EL APRENDIZAJE}

El alumnado encuestado demuestra cierto descuido, por parte del profesorado, para la realización de seminarios científicos. No obstante, valora la utilización del contrato pedagógico como método de evaluación, mostrando aplicaciones de la teoría a la práctica mediante estudios de casos y/o simulaciones; hace uso de la pregunta y la reflexión sistemática en el aula así como de una metodología variada y complementaria basada en el uso de las TIC. Así pues, el alumnado valora el que se establezca un entorno en el que el alumno ocupe un papel activo, ofreciéndole la oportunidad de realizar aportaciones personales con las que construir su propio conocimiento. De este modo, el alumnado percibe cómo el profesorado entiende el conocimiento como elemento necesario para interpretar la realidad y no sólo para superar una determinada materia.

Respecto a la evaluación, el alumnado afirma cómo el profesorado evalúa el proceso de enseñanza-aprendizaje mediante diversos métodos y procedimientos de evaluación continua con los que establecer propuestas de mejora. Además, hace uso de la tutoría académica mediante el diseño de un adecuado plan de trabajo.

Tabla 2. Análisis descriptivo para la escala "Modelo centrado en el aprendizaje"

\begin{tabular}{|l|c|c|}
\hline \multicolumn{1}{|c|}{ Ítem } & Media & Desv. Típica \\
\hline $\begin{array}{l}\text { El profesorado realiza seminarios con el alumnado de la } \\
\text { asignatura }\end{array}$ & 3.32 & 1.043 \\
\hline $\begin{array}{l}\text { El profesorado utiliza como método de evaluación el contrato } \\
\text { pedagógico, negociando con el alumnado, fijando tareas, los } \\
\text { trabajos que se deben elaborar, el tipo de examen, etc. }\end{array}$ & 3.58 & .930 \\
\hline $\begin{array}{l}\text { El profesorado muestra aplicaciones de la teoría a los } \\
\text { problemas reales }\end{array}$ & 3.67 & .904 \\
\hline $\begin{array}{l}\text { El profesorado evalúa no sólo para valorar los resultados } \\
\text { del alumno sino para obtener información del proceso de } \\
\text { aprendizaje e introducir las mejoras necesarias }\end{array}$ & 3.71 & .875 \\
\hline $\begin{array}{l}\text { El profesorado utiliza procedimientos de evaluación } \\
\text { formativa/continua revisando y devolviendo trabajos } \\
\text { corregidos al alumnado con instrucciones para la mejora }\end{array}$ & 3.71 & .931 \\
\hline $\begin{array}{l}\text { El profesorado hace uso de estudio de casos y/o simulaciones } \\
\text { para potenciar la integración de la teoría y la práctica }\end{array}$ & 3.74 & .878 \\
\hline $\begin{array}{l}\text { El profesorado utiliza la tutoría con un plan de trabajo } \\
\text { establecido de antemano, no limitándose a esperar a que los } \\
\text { alumnos acudan }\end{array}$ & 3.77 & 1.005 \\
\hline $\begin{array}{l}\text { El profesorado hace uso de la pregunta y la reflexión de } \\
\text { manera sistemática para ayudarnos a pensar }\end{array}$ & 3.79 & .871 \\
\hline $\begin{array}{l}\text { El profesorado adopta una metodología de enseñanza variada } \\
\text { y complementaria según las características del alumnado }\end{array}$ & 3.79 & .905 \\
\hline $\begin{array}{l}\text { El profesorado establece un entorno que moviliza el } \\
\text { aprendizaje activo del alumno }\end{array}$ & 3.83 & \\
\hline
\end{tabular}


Estudios Pedagógicos XLII, $N^{\circ}$ 1: 209-222, 2016

METODOLOGÍA DOCENTE Y DE EVALUACIÓN EN EL NUEVO ESPACIO DE CONVERGENCIA EUROPEA PARA LA FORMACIÓN DEL DOCENTE DE EDUCACIÓN SECUNDARIA

\begin{tabular}{|l|c|c|}
\hline $\begin{array}{l}\text { El profesorado entiende que los conocimientos adquiridos } \\
\text { por el alumnado le sirven para interpretar la realidad y no } \\
\text { sólo para aprobar la materia }\end{array}$ & 3.86 & .853 \\
\hline $\begin{array}{l}\text { El uso que el profesorado hace de las TIC fomenta la } \\
\text { participación, interactividad y cooperación del alumnado } \\
\text { mediante la tutoría telemática, foros de discusión, etc. }\end{array}$ & 3.90 & .918 \\
\hline $\begin{array}{l}\text { El profesorado complementa el examen como método de } \\
\text { evaluación con otros métodos de orientación formativa } \\
\text { (trabajos, ensayos, etc.) }\end{array}$ & 3.91 & .906 \\
\hline $\begin{array}{l}\text { El profesorado ofrece al alumnado la oportunidad de realizar } \\
\text { aportaciones personales (foros, tutorías, etc.) }\end{array}$ & 3.94 & .943 \\
\hline $\begin{array}{l}\text { Un buen profesor no presenta los conocimientos como } \\
\text { algo cerrado, sino como algo abierto a la reconstrucción y } \\
\text { elaboración personal del alumno }\end{array}$ & 4.05 & .850 \\
\hline
\end{tabular}

\subsection{HABILIDADES DOCENTES}

El alumnado se muestra indiferente al cuestionar la realización, por parte del profesorado, de una evaluación inicial con la que detectar sus conocimientos previos. No obstante, valora la síntesis que realiza el profesor sobre los aspectos a tratar al comienzo de las tutorías presenciales y a su término, estableciendo un clima de buenas relaciones interpersonales. Además, el profesorado realiza un seguimiento de los resultados de la evaluación para la planificación del proceso de enseñanza-aprendizaje, orientando y mejorando los resultados académicos según los objetivos previstos. Además, informa acerca de los métodos y criterios de evaluación así como los criterios de corrección de las pruebas.

El alumnado afirma cómo el profesorado se interesa por ellos a nivel personal, procurando trasmitirles su interés por la materia. Además, planifica su asignatura anualmente, estableciendo los objetivos, el programa de la asignatura, seleccionando los contenidos según criterios adecuados, utilizando variedad de recursos y referencias bibliográficas.

Tabla 3. Análisis descriptivo para la escala "Habilidades docentes"

\begin{tabular}{|l|c|c|}
\hline \multicolumn{1}{|c|}{ Ítem } & Media & Desv. Típica \\
\hline $\begin{array}{l}\text { El profesorado realiza una evaluación inicial para precisar los } \\
\text { conocimientos previos del alumnado }\end{array}$ & 3.20 & 1.242 \\
\hline $\begin{array}{l}\text { Al terminar las tutorías presenciales, el profesorado hace una } \\
\text { breve síntesis de lo tratado en ella }\end{array}$ & 3.52 & .974 \\
\hline $\begin{array}{l}\text { Al comenzar las sesiones de tutorías presenciales, el profe- } \\
\text { sorado recuerda brevemente lo tratado en la sesión anterior }\end{array}$ & 3.54 & 1.085 \\
\hline $\begin{array}{l}\text { El profesorado tiene en cuenta los resultados de la evaluación } \\
\text { para modificar su planificación, metodología y actividad } \\
\text { docente a corto o medio plazo }\end{array}$ & 3.56 & .974 \\
\hline
\end{tabular}


Estudios Pedagógicos XLII, N ${ }^{\circ}$ : 209-222, 2016 METODOLOGÍA DOCENTE Y DE EVALUACIÓN EN EL NUEVO ESPACIO DE CONVERGENCIA EUROPEA PARA LA FORMACIÓN DEL DOCENTE DE EDUCACIÓN SECUNDARIA

\begin{tabular}{|l|c|c|}
\hline $\begin{array}{l}\text { El profesorado evalúa en diferentes momentos del curso para } \\
\text { llevar un seguimiento del aprendizaje del alumnado }\end{array}$ & 3.58 & 1.006 \\
\hline El profesorado se interesa por los estudiantes como personas & 3.69 & 1.024 \\
\hline $\begin{array}{l}\text { El profesorado da a conocer las referencias bibliográficas } \\
\text { esenciales para la materia }\end{array}$ & 3.72 & .909 \\
\hline $\begin{array}{l}\text { El profesorado planifica su asignatura todos los cursos } \\
\text { dedicando tiempo a esta tarea }\end{array}$ & 3.72 & .858 \\
\hline $\begin{array}{l}\text { El profesorado comunica al alumnado los objetivos de cada } \\
\text { uno de los temas a tratar }\end{array}$ & 3.76 & .926 \\
\hline $\begin{array}{l}\text { El profesorado utiliza variedad de recursos (audiovisuales, } \\
\text { transparencias, vídeo, pizarra, documentos, etc.) que facilitan } \\
\text { la presentación de los contenidos }\end{array}$ & 3.77 & .918 \\
\hline $\begin{array}{l}\text { El profesorado orienta al alumnado para que mejore sus } \\
\text { resultados }\end{array}$ & 3.78 & .984 \\
\hline $\begin{array}{l}\text { El profesorado selecciona los contenidos que se va a impartir } \\
\text { utilizando criterios adecuados (objetivos, relevancia, utilidad, } \\
\text { interés, etc.) }\end{array}$ & 3.80 & .848 \\
\hline $\begin{array}{l}\text { El profesorado evalúa los aprendizajes de acuerdo con los } \\
\text { objetivos establecidos en la planificación }\end{array}$ & 3.81 & .850 \\
\hline $\begin{array}{l}\text { El profesorado procura transmitir al alumnado su interés por } \\
\text { la materia }\end{array}$ & 3.83 & .877 \\
\hline $\begin{array}{l}\text { Conozco los criterios de corrección de las pruebas que el } \\
\text { profesorado utiliza }\end{array}$ & 3.86 & .983 \\
\hline $\begin{array}{l}\text { El profesorado procura que en las sesiones de tutorías } \\
\text { presenciales exista un clima de buenas relaciones } \\
\text { interpersonales }\end{array}$ & 3.86 & .896 \\
\hline $\begin{array}{l}\text { El profesorado establece con claridad los criterios de } \\
\text { evaluación del aprendizaje del alumnado }\end{array}$ & 3.86 & 3.85 \\
\hline $\begin{array}{l}\text { El profesorado informa al alumnado de los métodos de } \\
\text { evaluación a utilizar }\end{array}$ & 3.88 \\
\hline $\begin{array}{l}\text { El profesorado establece claramente los objetivos de su } \\
\text { asignatura }\end{array}$ & 3.92 & .93 \\
\hline $\begin{array}{l}\text { El profesorado facilita el programa de la asignatura e informa } \\
\text { sobre el mismo }\end{array}$ & 3.93 & \\
\hline
\end{tabular}

A continuación, se analiza la relación entre los factores que agrupan cada escala del cuestionario mediante la correlación $\mathrm{r}$ de Pearson. Para la primera escala "Modelo centrado en la enseñanza", los resultados demuestran cómo existe correspondencia entre las percepciones del alumnado hacia la concepción tradicional del conocimiento y el aprendizaje en dicho título de posgrado, y la concepción tradicional de la enseñanza y el papel del profesor en el aula. De igual modo, se comprueba reciprocidad entre la concepción tradicional del conocimiento y de la enseñanza, y el uso de métodos de enseñanza y evaluación de carácter tradicional. 
Estudios Pedagógicos XLII, Nº 1: 209-222, 2016

METODOLOGIA DOCENTE Y DE EVALUACIÓN EN EL NUEVO ESPACIO DE CONVERGENCIA EUROPEA PARA LA FORMACIÓN DEL DOCENTE DE EDUCACIÓN SECUNDARIA

Tabla 4. Correlaciones entre factores para la escala "Modelo centrado en la enseñanza"

\begin{tabular}{|l|c|c|c|}
\hline & \multicolumn{3}{|c|}{ r } \\
\cline { 2 - 5 } & F1 & F2 & F3 \\
\hline F1. Concepción tradicional del conocimiento y del aprendizaje & & $.542^{* *}$ & $.460^{* *}$ \\
\hline F2. Concepción tradicional de la enseñanza y del papel del profesor & $.542^{* *}$ & & $.353^{* *}$ \\
\hline F3. Uso de métodos de enseñanza y evaluación tradicionales & $.460^{* *}$ & $.353^{* *}$ & \\
\hline
\end{tabular}

** La correlación es significativa al nivel .01 (bilateral)

Tomando como referencia la escala "Modelo centrado en el aprendizaje", los resultados apuntan a la existencia de relación entre las percepciones del alumnado del Máster hacia la concepción activa y constructiva de la enseñanza y la actuación coherente del profesor universitario como mediador, y la concepción constructivista del aprendizaje. De igual modo, existe reciprocidad entre las percepciones del alumnado universitario hacia la concepción activa y constructivista de la enseñanza y del aprendizaje, y la interacción eficaz con los estudiantes, así como con el uso de metodologías de evaluación de tipo formativo.

Tabla 5. Correlaciones entre factores para la escala "Modelo centrado en el aprendizaje"

\begin{tabular}{|l|c|c|c|c|}
\hline & \multicolumn{4}{|c|}{ r } \\
\cline { 2 - 5 } & F1 & F2 & F3 & \multicolumn{1}{|c|}{ F4 } \\
\hline $\begin{array}{l}\text { F1. Concepción activa y constructiva de la enseñanza } \\
\text { y actuación coherente }\end{array}$ & & $.742^{* *}$ & $.682^{* *}$ & $.695^{* *}$ \\
\hline $\begin{array}{l}\text { F2. El conocimiento como construcción. Concepción } \\
\text { constructivista del aprendizaje }\end{array}$ & $.742^{* *}$ & & $.453^{* *}$ & $.552^{* *}$ \\
\hline $\begin{array}{l}\text { F3. Interacción eficaz con los estudiantes, en tutorías, } \\
\text { seminarios, uso de las TICs, etc. }\end{array}$ & $.682^{* *}$ & $.453^{* *}$ & & $.675^{* *}$ \\
\hline $\begin{array}{l}\text { F4. Uso de metodologías de evaluación de tipo } \\
\text { formativo }\end{array}$ & $.695^{* *}$ & $.552^{* *}$ & $.675^{* *}$ & \\
\hline
\end{tabular}

** La correlación es significativa al nivel .01 (bilateral)

Finalmente, y para la escala "Habilidades docentes", se aprecian correlaciones entre las percepciones del alumnado hacia las habilidades docentes, para planificar e informar sobre la asignatura, y las habilidades de manejo instruccional (contenidos, recursos, etc.). Además, existe reciprocidad entre éstas y las habilidades de interacción y/o relación educativa y evaluación (evaluación acorde con los objetivos establecidos en la fase de planificación y evaluación inicial, continua y formativa). 
Tabla 6. Correlaciones entre factores para la escala "Habilidades docentes"

\begin{tabular}{|l|c|c|c|c|c|}
\hline & \multicolumn{5}{|c|}{ r } \\
\cline { 2 - 6 } & F1 & F2 & F3 & F4 & F5 \\
\hline $\begin{array}{l}\text { F1. Habilidades de planificación/infor- } \\
\text { mación a los estudiantes }\end{array}$ & & $.730^{* *}$ & $.699^{* *}$ & $.741^{* *}$ & $.480^{* *}$ \\
\hline F2. Habilidades de manejo instruccional & $.730^{* *}$ & & $.750^{* *}$ & $.704^{* *}$ & $.657^{* *}$ \\
\hline $\begin{array}{l}\text { F3. Habilidades de interacción/relación ed- } \\
\text { ucativa }\end{array}$ & $.699^{* *}$ & $.750^{* *}$ & & $.772^{* *}$ & $.601^{* *}$ \\
\hline $\begin{array}{l}\text { F4. Habilidades de evaluación. Evaluación } \\
\text { acorde con objetivos, con criterios explíci- } \\
\text { tos y con información a los estudiantes }\end{array}$ & $.741^{* *}$ & $.704^{* *}$ & $.772^{* *}$ & & $.560^{* *}$ \\
\hline F5. Habilidades de evaluación & $.480^{* *}$ & $.657^{* *}$ & $.601^{* *}$ & $.560^{* *}$ & \\
\hline
\end{tabular}

** La correlación es significativa al nivel .01 (bilateral)

\section{DISCUSIÓN Y CONCLUSIONES}

Este estudio ha permitido conocer las percepciones del alumnado del Máster en formación del profesorado de Educación Secundaria Obligatoria acerca de la metodología docente y evaluativa desarrollada por el profesorado que imparte docencia en dicho título. Así pues, el Espacio Europeo de Educación Superior ha supuesto la definición de un nuevo modelo educativo que requiere de un perfil profesional, roles y actividades diferentes a las tradicionales en estudiantes y profesorado de Educación Superior; todo ello implica "aprender de otro modo" y también "enseñar de otro modo" (Fernández, 2006).

Los resultados obtenidos demuestran cómo, para el modelo centrado en la enseñanza, el profesorado no sólo utiliza el examen como instrumento de evaluación. Además entiende que un buen profesor es aquel que explica adecuadamente su asignatura y domina la materia que imparte. Las percepciones del alumnado hacia la concepción tradicional del conocimiento guardan relación con sus valoraciones acerca de la concepción tradicional de la enseñanza y el papel que ocupa el profesor universitario en dicho ámbito, así como con el uso de métodos de enseñanza y evaluación tradicionales. Estos resultados se relacionan con los aportados por Torres, Ruiz y Álvarez (2007), quienes inciden en la necesidad de que el profesorado disponga de suficientes conocimientos de la materia que imparte y el dominio de la pedagogía; además, Manso (2005) define al profesor universitario como gestor de las actividades de aprendizaje, con suficiente formación psicopedagógica y práctica investigadora sobre su propia docencia.

Para el modelo centrado en el aprendizaje, el alumnado valora el que el profesorado realice aplicaciones de la teoría a la práctica en el aula, diseñe un plan de trabajo a desarrollar en las tutorías presenciales, fomente la participación del alumnado, así como un sistema de evaluación formativa que tenga en cuenta no sólo el resultado final, sino el proceso de enseñanza-aprendizaje a desarrollar. Además, se establecen relaciones entre las percepciones del alumnado del Máster hacia la concepción activa y constructivista de la enseñanza y el aprendizaje, la interacción de éste con los estudiantes y el uso de un sistema de evaluación de tipo formativo. 
Estos resultados contradicen los aportados por Hernández-Amorós y Carrasco (2012) quienes revelan cómo el alumnado del Máster de Secundaria se muestra disconforme con la vinculación entre la teoría y la práctica en el desarrollo de programas formativos y la coordinación entre el profesorado. No obstante, y tal y como apuntan Palazón et al. (2011), y Rodríguez-Martín y Álvarez-Arregui (2013), el alumnado se decanta hacia el desarrollo de metodologías activas y participativas para favorecer la motivación, el desarrollo de proyectos y la autogestión en el trabajo individual y en equipo.

Respecto a las habilidades docentes, el alumnado aprecia que el profesor realice una síntesis de lo tratado en las tutorías, planifique su asignatura adecuadamente y se interese por él no sólo a nivel académico, sino también personal. Además, se aprecia reciprocidad en las valoraciones del alumnado hacia las habilidades de planificación y manejo instruccional del profesorado, y sus habilidades de interacción y/o relación educativa y de evaluación del proceso de enseñanza-aprendizaje. Estos resultados avalan algunas de las competencias para el desarrollo de una función docente de calidad expuestas por Mas (2011), entre las que se destaca el diseño de la guía académica de la asignatura según las necesidades, contextos y el perfil profesional al que pertenece.

Así pues, las percepciones del alumnado hacia la metodología docente y evaluativa debe hacer reflexionar al profesorado universitario de los procedimientos para enseñar y evaluar en el nuevo espacio construido en la Educación Superior, gracias a la implantación del Espacio Europeo de Educación Superior. Dado que el proceso de convergencia europea apuesta por una formación integral a lo largo de la vida del alumnado, este trabajo analiza el proceso de enseñanza-aprendizaje en el alumnado de posgrado, pues incide de manera directa en la calidad del sistema universitario español y, más concretamente, en su rendimiento académico.

No obstante, la escasa muestra con la que se ha llevado a cabo dicha investigación no nos permite garantizar la generalización de los resultados a otras muestras. Además, el uso exclusivo del cuestionario como instrumento de recogida de datos puede generar problemas de deseabilidad social y sinceridad al cumplimentarlo. Por ello, puede resultar interesante conocer las valoraciones del profesorado y, de este modo, contrastar la información obtenida sobre el modo del proceder del profesorado en el aula universitaria. Así pues, este trabajo puede servir para realizar un primer diagnóstico de las concepciones del profesorado del Máster en Formación del Profesorado de la Universidad Católica de Murcia en torno al conocimiento, el aprendizaje, la enseñanza y la evaluación analizando, además, el modo de concretar dichas concepciones en su trabajo en el aula universitaria. Además, es válido para analizar las habilidades docentes en el ámbito de la Educación Superior. Incluso, se puede analizar las percepciones del alumnado para el título de Grado, estableciendo una comparativa entre alumnado de Posgrado y Grado y así poder comprender sus necesidades e inquietudes respecto a la metodología del profesorado universitario.

\section{REFERENCIAS BIBLIOGRÁFICAS}

Armengol, C., Castro, D., Durán, Mª M., Essomba, M. Á., Feixas, M., Gairín, J., Navarro, M. y Tomas, M. (2009). La coordinación académica en la Universidad. Estrategias para una educación de calidad. Revista Electrónica Interuniversitaria de Formación del Profesorado, vol. 12, n. 2, $121-144$.

Cano, M ${ }^{a}$ E. (2008). La evaluación por competencias en la educación superior. Profesorado, revista 
de currículum y formación del profesorado, vol. 12, n. 3, 220-235, en: www.ugr.es/ recfpro/ rev123COL1.pdf (consulta: 2 de Abril de 2014).

Carrasco, A., Donoso, A., Duarte, T., Hernández, J.J., López, R. y Núñez, C. (2010). La utilización de metodologías activas de aprendizaje en la enseñanza de la contabilidad. Libro de actas del XIV Encuentro de la Asociación Española de Profesores Universitarios de Contabilidad. La Coruña.

Cid, A., Zabalza, M. A. y Doval, M I. (2012). La docencia universitaria: un modelo para su análisis. Revista de Docencia Universitaria, vol. 10, n. 1, 87-104, en: http://red-u.net/redu/index.php/ REDU/article/view/315 (consulta: 2 de Abril de 2014).

Fernández, $M^{a}$ A. (2006). Metodologías activas para la formación de competencias. Educatio siglo $X X I$, vol. 24, 35-56.

Gairín, J., Feixas, M., Guillamón, C. y Quinquer, D. (2004). La tutoría académica en el escenario europeo de la Educación Superior. Revista Interuniversitaria de Formación del Profesorado, vol. 18, n. 1, 61-77, en http://ww.aufop.com/aufop/uploaded_files/articulos/1219255665.pdf (consulta: 1 de Abril de 2014).

Gargallo, B., Suárez, J. y Ferreras, A. (2007). Estrategias de aprendizaje y rendimiento académico en estudiantes universitarios. Revista de Investigación Educativa, vol. 25, n. 2, 421-441, en: http:// revistas.um.es/rie/article/view/96941 (consulta: 1 de Abril de 2014).

Gargallo, B., Suárez, J., Gargella, P. y Fernández, A. (2011). El cuestionario CEMEDEPU. Un instrumento para la evaluación de la metodología docente y evaluativa de los profesores universitarios. Estudios sobre Educación, vol. 21, 9-40, en: http://dspace.unav.es/dspace/ handle/10171/22511 (consulta: 3 de Abril de 2014).

González, N. y García, Mª R. (2007). El aprendizaje cooperativo como estrategia de enseñanza aprendizaje en psicopedagogía (UC): repercusiones y valoraciones de los estudiantes. Revista Iberoamericana de Educación, vol. 42, n. 6, 1-13, en: http://www.rieoei.org/expe/1723Fernandez. pdf (consulta: 1 de Abril de 2014).

Guzmán, J. C. (2011). La calidad de la enseñanza en educación superior ¿Qué es una buena enseñanza en este nivel educativo? Perfiles educativos, vol. 33, 129-141.

Hernández-Amorós, M ${ }^{\mathrm{a}}$ J. y Carrasco, V. (2012). Percepciones de los estudiantes del Máster en Formación del Profesorado de Educación Secundaria: fortalezas y debilidades del nuevo modelo formativo. Enseñanza \& Teaching, vol. 30, n. 2, 127-152, en: http://dialnet.unirioja.es/servlet/art iculo? codigo $=4091444 \&$ orden $=373268 \&$ info=link (consulta: 3 de Abril de 2014).

Hernández-Pina, F. (2003). Consistencia entre motivos y estrategias de aprendizaje en estudiantes universitarios. Revista de Investigación Educativa, vol. 20, 487-510, en: http://revistas.um.es/ rie/article/view/99031 (consulta: 3 de Abril de 2014).

Herrera, L., Lorenzo, O. y Rodríguez, C. (2008). Las tutorías en el Espacio Europeo de Educación Superior: valoración de su implementación en la titulación de Maestro. Revista de Investigación Psicoeducativa, vol. 6, n. 1, 65-85.

Herrera, L. (2007). Experiencia Piloto de Implantación del Sistema de Transferencia de Créditos Europeos (ECTS) en la Titulación de Maestro. Valoración del profesorado y el alumnado participante. En Rosabel, R. (Dir.). Investigar el cambio curricular en el Espacio Europeo de Educación Superior (pp. 159-178). Alicante: Editorial Marfil.

Herrera, L. y Cabo. J.M. (2008). Experiencias piloto de implantación del sistema europeo de transferencia y acumulación de créditos (ECTS). Reflexiones derivadas de su aplicación práctica en diferentes universidades españolas. Granada: Editorial Comares.

Ion, G. y Cano, E. (2011). El proceso de implementación de la evaluación por competencias en la Educación Superior. Un estudio sobre el rol de los cargos académicos. Revista de Investigación en Educación, vol. 9, n. 2, 246-258, en: http://webs.uvigo.es/reined/ojs/index.php/reined/article/ viewFile/304/172 (consulta: 1 de Abril de 2014).

Kallioinen, O. (2010). Defining comparing generic competencies in Higher Education. European Educational Research Journal, vol. 9, n. 1, 6-68. 
Estudios Pedagógicos XLII, N 1: 209-222, 2016

METODOLOGİA DOCENTE Y DE EVALUACIÓN EN EL NUEVO ESPACIO DE CONVERGENCIA EUROPEA PARA LA FORMACIÓN DEL DOCENTE DE EDUCACIÓN SECUNDARIA

Manso, J.M. (2005). Reflexiones sobre un nuevo modelo de profesor universitario en Medicina. Una visión crítica de la enseñanza médica. En Actas del $2^{\circ}$ Congreso Virtual de Cardiología, en: http://www.fac.org.ar/scvc/llave/edu/manso/mansoe.htm (consulta: 10 de Junio de 2014).

Martínez, B., García, J.N., Robledo, P., Díez, C., Álvarez, Ma L., Marbán, J., Fuertes, A. Mª., Fidalgo, R., Arias, O., Pacheco, D. y Rodríguez, C. (2007). Valoración docente de las metodologías activas: Un aspecto clave en el proceso de convergencia europea. Aula Abierta, vol. 35, n. 1, 49-62, en: http://redined.mecd.gob.es/xmlui/handle/11162/4848 (consulta: 2 de Junio de 2014).

Mas, O. (2011). El profesor universitario: sus competencias y formación. Profesorado. Revista de curriculum y formación del profesorado, vol. 15, n. 3, 195-211, en: http: //www.ugr.es/local/ recfpro/rev153COL1.pdf (consulta: el 2 de Junio de 2014).

Medina, A., Domínguez, Mª C. y Ribeiro, F. (2011). Formación del profesorado universitario en las competencias docentes. Revista de Historia de la Educación latinoamericana, vol. 13, n. 17, 119-138, en: http://www.redalyc.org/articulo.oa?id=86922615006 (consulta: 3 de Junio de 2014).

Méndez, C. (2005). La implantación del sistema de créditos europeo como una oportunidad para la innovación y mejora de los procedimientos de enseñanza aprendizaje en la universidad. Revista Española de Pedagogía, vol. 63, n. 230, 43-62.

Moreno, S., Bajo, Ma T., Moya, M., Maldonado, A. y Tudela, P. (2007). Las competencias en el nuevo paradigma educativo para Europa. Granada: Vicerrectorado de Planificación, Calidad y Evaluación Docente de la Universidad de Granada.

Padilla, Ma T. y Gil, J. (2008). La evaluación orientada al aprendizaje en la Educación Superior: condiciones y estrategias para su aplicación en la docencia universitaria. Revista Española de Pedagogía, vol. 241, 467-486, en: http://evaluacion.webatu.com/web_documents/lectura_no6. pdf (consulta: 4 de Abril de 2014).

Palazón, A., Gómez, M. Gómez, J.C., Pérez, M. y Gómez, J. (2011). Relación entre la aplicación de metodologías docentes activas y el aprendizaje del estudiante universitario. Bordón. Revista de pedagogía, vol. 63, n. 2, 27-40, en: http://dialnet.unirioja.es/servlet/articulo?codigo=3657074 (consulta: 7 de Abril de 2014).

Palomares, A. (2011). El modelo docente universitario y el uso de nuevas metodologías en la enseñanza, aprendizaje y evaluación. Revista de Educación, vol. 355, 591-604, en: http://www. revistaeducacion.educacion.es/re355_25.html (consulta: 7 de Abril de 2014).

Pozo, J. I. y Pérez, M ${ }^{a}$ P. (2009). Psicología del aprendizaje universitario: la formación en competencias. Madrid: Morata.

Rodríguez-Martín, A. y Álvarez-Arregui, E. (2013). Metodologías y recursos en las titulaciones de Grado: perspectivas de estudiantes y responsables institucionales. Revista Electrónica de Formación del Profesorado, vol. 16, n. 2, 105-120, en: http://revistas.um.es/reifop/article/ view/180861 (consulta: 3 de Abril de 2014).

Sáez, J. M. y Ruiz, J. M. (2012). Metodología didáctica y tecnología educativa en el desarrollo de las competencias cognitivas: aplicación en contextos universitarios. Profesorado. Revista de Curriculum y formación del profesorado, vol. 16, n. 3, 373-391, en: http://www.ugr.es/local/ recfpro/rev163COL9.pdf (consulta: el 1 de Abril de 2014).

Sander, P. (2005). La investigación sobre nuestros alumnos en pro de una mayor eficacia en la enseñanza universitaria. Electronic Journal of Research in Educational Psychology, vol. 3, n. 1, 113-130.

Tirado, F., Miranda, A. y Sánchez, A. (2007). La evaluación como proceso de legitimidad: la opinión de los alumnos. Reporte de una experiencia. Perfiles educativos, vol. 29, n. 118, 7-24.

Torres, A., Ruiz, J.C. y Álvarez, N. (2007). La auto-transformación del estudiante universitario: más allá de la formación integral. Revista Iberoamericana de Educación, vol. 43, n. 4, 1-9, en: http:// www.rieoei.org/deloslectores/1792Torres.pdf (consulta: 5 de Abril de 2014).

Zabalza, M. A. (2012). El estudio de las "buenas prácticas" docentes en la enseñanza universitaria. Revista de Docencia Universitaria, vol. 10, n. 1, 17-42, en: http://red-u.net/redu/index.php/ REDU/article/view/363 (consulta: 6 de Abril de 2014). 